\section{Optimization of nutritional and physicochemical parameters for prodiogiosin production by Serratia nematodiphila}

\author{
Chandrima Ghosh, Shraddha Patel* \\ Department of Microbiology, Rayat Shikshan Sanstha’s Karmaveer Bhaurao Patil College (K.B.P.), \\ Vashi, Navi Mumbai 400703, India \\ ${ }^{\star}$ Corresponding author, E-mail: gati_1982@yahoo.com
}

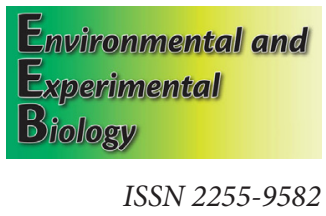

UNIVERSITY OF LATVIA

\begin{abstract}
The increasing interest in a sustainable approach to biotechnological applications has tremendously invigorated the scope of natural pigments. In the present study, bacterial cultures were screened from environmental samples, and Serratia nematodiphila was identified as a potential prodigiosin producer. The pigment was characterized as prodigiosin on the basis of a presumptive acid test and observation of absorption maxima of acidified pigment at $535 \mathrm{~nm}$. In order to increase prodigiosin production from the test isolate, various nutritional and physicochemical parameters were optimized. Experiments revealed that nutrient broth medium supplemented with $0.2 \%$ mannitol and $0.2 \%$ inorganic phosphate supported high pigment production. Optimum prodigiosin production was achieved when the nutrient broth media (with $1 \% \mathrm{NaCl}$ ) was adjusted to $\mathrm{pH} 8$ and incubated at $25^{\circ} \mathrm{C}$ for $48 \mathrm{~h}$. The optimized media showed over 64.78 and $73.23 \%$ increase in prodigiosin production as compared to the nutrient broth and Luria Bertani broth, respectively. The present study indicated a great potential of $S$. nematodiphila to produce prodigiosin by minimum optimization of culture medium.
\end{abstract}

Key words: optimization, pigment, prodigiosin, Serratia nematodiphila, sustainable production.

Abbreviations: NA, nutrient agar; NB, nutrient broth.

\section{Introduction}

Colour and texture significantly contribute to the visual appeal of finished goods. Although the texture of products is primarily dependent on critical manufacturing processes, it is ultimately the colour that determines the acceptance of a product in the market. Therefore, many artificial synthetic colourants find extensive applications in food, pharmaceutical, textile and cosmetic industries (Kumar et al. 2017). Many of these synthetic pigments, however, pose a serious threat to the environment and can cause severe damage to living beings (Duran et al. 2002). Most commonly, these artificial pigments cause allergies, respiratory distress and cancer in humans (Kobylewski, Jacobson 2012). As a result, many synthetic dyes are banned from production and use based on their toxicity profile. As a result, the need for a sustainable production approach of environmentaly-safe pigments has increased considerably to meet the industrial demand (Babu, Shenolikar 1995).

Natural pigments are obtained mainly from plants or microorganisms (Yusuf et al. 2017). Although plant sources of pigments are viable options, they suffer from numerous drawbacks like instability to heat, light and $\mathrm{pH}$, and low solubility as compared to microbial pigments (Kim et al. 1999). Moreover, in order to meet the industrial demand, valuable resources and land will be required for its cultivation. This can negatively affect the food and agriculture sector (Purnama et al. 2017). In contrast, the microbial sources of pigments offer several advantages like fast growth rate, use of economical raw material and availability throughout the year (Narsing Rao et al. 2017). Furthermore, there is an abundance of microorganisms in nature (fungi, yeasts, and bacteria) producing pigments like carotenoids, melanins, flavins, quinones and prodigiosins (Duffose 2009). Biosynthesized pigments can also serve as major chromophores, which, on further chemical modification, provide a broader spectrum of colours (Hobson et al. 1998). Thus, diverse shades of colour can be obtained from pigments produced by microorganisms, which are beneficial in the food, textile and cosmetic industries.

Common environmental isolates of bacteria of the genus Serratia are known to produce various secondary metabolites including a tripyrrole pigment prodigiosin (Duffose 2009). Pigments of the prodigiosin family are particularly in demand by various industries due to their remarkable and diverse range of biological effects (Venil, Lakshmanaperumalsamy 2009). They have been widely studied for cytotoxic activity (Furstner 2003; Darshan, Manonmani 2015). In addition, these pigments also act as potential antioxidants and antimicrobial agents (Lewis, Corpe 1964). 
Colour stability under extremes of $\mathrm{pH}$, temperature and other processing conditions is a prerequisite for the production of a natural pigment for commercial use. Relevant literature is available on pigment production and optimization. However, more in-depth studies are required to further enhance our understanding to support a sustainable pigment industry. In respect to prodigiosin production, both strain as well as species dependent variation in prodigiosin biosynthesis gene cluster was demonstrated (Harris et al. 2004). Moreover, every strain of bacteria is unique in its nutritional requirement for production of specific secondary metabolites, including pigments. For instance, a strain of Serratia nematodiphila RL2 utilized 1\% lactose and $1 \%$ yeast extract as carbon and nitrogen sources, respectively, and metal-containing chemical factors such as uranyl acetate for optimum pigment production (Gondil et al. 2017). Moreover, certain metabolites require a complex media that is rich in a variety of nutrients, whereas others require nutrient-limiting conditions for their production. For example, a Serratia marcescens strain was observed to utilize fatty acids to produce prodigiosin pigment, whereas a soft rot fungus Chlorociboria aeruginascens required nitrogen-limiting conditions for production of a blue-green pigment xylindein (Stange et al. 2019). For these reasons, the optimization of pigment production is a crucial process to identify the factors that specifically promote or limit its production. These factors include media composition, carbon and nitrogen sources, as well as physicochemical parameters like $\mathrm{pH}$, temperature, incubation conditions, initial culture density and aeration (Wei et al. 2005). The presence of specific micronutrients like iron and sea salts (Silverman, Munoz 1973) and glycerol (Williams, Green 1956) also affects pigment production, and hence should be evaluated with respect to efficient productivity from bacteria.

The aim of the present study was to optimize various growth parameters for efficient production of prodigiosin by a particular soil isolate of $S$. nematodiphila.

\section{Materials and methods}

\section{Sample collection}

Environmental samples like garden soil and leaf swabs were collected from K.B.P College, Vashi, India. Air sampling in the microbiology laboratory in K.B.P College was also carried out. Other soil samples were collected from the textile industrial area, petrol pumps and dumping grounds in Kalyan, Ulhasnagar, Shahad and Titwala. In addition, a mangrove sample was collected from Mahim creek, and another soil sample was obtained from West Bengal.

\section{Isolation and screening of pigment producers}

The isolation and screening of pigment producing organisms from the above samples were carried out on nutrient agar (NA) medium ( $\mathrm{pH} 7$ ). The obtained pigment producers were re-isolated on NA plates to confirm their purity and single colonies were streaked on NA slants and stored at $4{ }^{\circ} \mathrm{C}$ until further use.

\section{Presumptive color tests for prodigiosin}

The presumptive test for characterization of prodigiosin was carried out as indicated by Gerber and Lechevalier (1976). The pigmented colonies obtained on NA plates were suspended in saline and re-isolated on sterile plates to confirm the purity of the isolate. The growth obtained after $48 \mathrm{~h}$ was scraped from the agar plates and suspended overnight in $95 \%$ ethanol at room temperature. The suspension was centrifuged at $5000 g_{\mathrm{n}}$ for $15 \mathrm{~min}$ to remove the cell debris and the clear solution was acidified with a drop of concentrated hydrochloric acid. A red or pink color in the acidified solution indicated a positive, presumptive test for prodigiosin.

\section{Identification of the isolate}

Preliminary identification of the prodigiosin producing isolate was performed based on the observed colony characteristics that defined the cultural and morphological properties. The biochemical characteristics were studied further and classified by comparison of the obtained results with standard tests (Holt et al. 1994). The results of preliminary identification were confirmed with 16s rRNA characterization that was carried out at Chromous Biotech, Hyderabad.

\section{Characterization of prodigiosin based on UV-visible spectrophotometry}

Prodigiosin displays a characteristic absorption spectrum in acidified methanol, with a strong absorption peak at $\lambda_{\max } 535$ $\mathrm{nm}$. Hence, in order to confirm prodigiosin production the pigment producing cultures showing a positive presumptive test were grown in nutrient broth (NB) for $24 \mathrm{~h}$ at $30^{\circ} \mathrm{C}$. A $1 \mathrm{~mL}$ volume of sample was harvested and centrifuged at $13000 \mathrm{rpm}$ for $5 \mathrm{~min}$. The supernatant was discarded and the pellet was re-suspended in acidified ethanol ( $4 \% 1 \mathrm{M}$ $\mathrm{HCl}$ in ethanol) to extract prodigiosin from the cells. Cell debris was removed by a second centrifugation step, and the supernatant was transferred to a cuvette (Hardjito et al. 2002). The absorption spectrum was measured using a UVvisible spectrophotometer Systronics 2203.

\section{The quantification of prodigiosin production}

The pigment producing cells were grown and harvested as described above. The cell free supernatant was subjected to spectral scanning in the range of 300 to $700 \mathrm{~nm}$. The prodigiosin production was quantified using the following equation, and expressed as relative concentration of prodigiosin produced per cell (Slater et al. 2003):

Prodigiosin unit $=\left[\mathrm{OD}_{499}-\left(1.381 \times \mathrm{OD}_{620}\right)\right] / \mathrm{OD}_{620}$ $\times 1000$,

where $\mathrm{OD}_{499}$ is pigment absorbance at $499 \mathrm{~nm}, \mathrm{OD}_{620}$ is bacterial cell absorbance at $620 \mathrm{~nm}$ and 1.381 is a constant. 


\section{Optimization of nutritional and physicochemical parameters for prodigiosin production}

Different environmental parameters have significant effect on prodigiosin production in the culture medium. Considering the complexity of mineral medium, its use was avoided in our study. Instead, basic NB medium that supported good growth of isolates was selected for optimization studies. The optimization of parameters was performed by using one factor at a time approach. In this technique, one parameter was varied at a time while keeping the others constant. To study different parameters, $0.5 \mathrm{~mL}$ of culture suspension was inoculated in NB and incubated at room temperature $\left(30^{\circ} \mathrm{C}\right)$ for $48 \mathrm{~h}$. The physicochemical parameters studied were $\mathrm{pH}(5,6,7,8)$, temperature $(25$, $\left.30,37,45,55^{\circ} \mathrm{C}\right), \mathrm{NaCl}$ concentration $(0.5,1,2,2.5 \%)$ and aeration (static or shaker) condition (Heinemann et al. 1970; Qadri, Williams 1972; Rjazantseva et al. 1994; Sole et al. 1994).

The nutritional parameters were studied using different carbon ( $0.2 \%$ lactose, xylose, mannitol and glucose) and nitrogen (yeast extract, peptone, tryptophan, beef extract and ammonium sulphate) sources (Wei, Chen 2005). In addition, the effect of various amino acids $(0.1 \%$ threonine, lysine, leucine, tryptophan, tyrosine, glutamate, glutamine, aspartate, asparagine, proline, phenylalanine, cysteine, isoleucine, arginine, methionine, alanine, histidine, serine, valine, and glycine) and phosphate sources $\left(\mathrm{CaSO}_{4}\right.$, $\mathrm{NaH}_{2} \mathrm{PO}_{4}, \mathrm{Co}_{3}\left(\mathrm{PO}_{4}\right)_{2}$ and $\mathrm{K}_{2} \mathrm{HPO}_{4}$ ) was also studied (Qadri, Williams 1973; Lawanson, Sholeye 1976; Cang et al. 2000; Slater et al. 2003).

The effect of amino acids was first checked qualitatively by disk diffusion method. Shortly, the sterilized filter paper discs were dipped in the above mentioned amino acid solutions (0.1\%) and placed on M9 minimal salt agar medium (with $0.5 \%$ glucose) swabbed with test isolate. The amino acids supporting pigmentation were further studied to estimate the exact concentration required for optimized pigment production.

To determine efficiency of prodigiosin production in optimized media, it was compared with two standard media, i.e., NB and Luria Bertani broth.

\section{Statistical analysis}

All the readings were carried out in sets of triplicates and the results were reported as mean \pm standard deviation.

Table 1. Pigment-producing bacteria isolated in the present study

\begin{tabular}{lll} 
Sample No. & Color & Sample source \\
S01A & Bright orange & Air \\
\hline S02G & Dull orange & Garden soil \\
S03U & Pinkish red & Ulhas river \\
S04K & Magenta & Kalyan soil \\
S05W & Orange & West Bengal soil \\
S06M & Orange & Mangrove sample
\end{tabular}

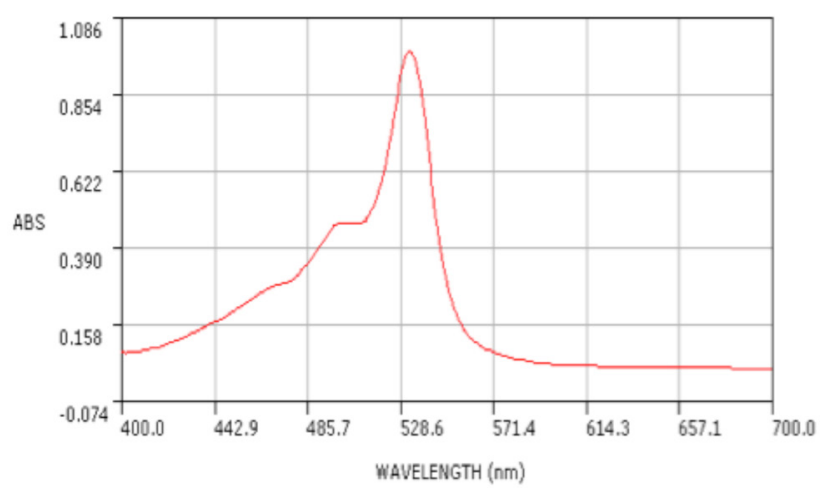

Fig. 1. Absorption of prodigiosin showing a peak at $535 \mathrm{~nm}$.

The distribution of variables was evaluated by one-way ANOVA to assess the significance of differences between mean values at $95 \%$ confidence interval $(p<0.05)$.

\section{Results}

Six pigment-producing bacterial isolates were obtained in our study. The colour and source of these isolates are shown in Table 1. Distinct shades of orange to magenta red colonies were observed on NA plates. Among these, the presumptive test for prodigiosin was positive only for the pigment produced by isolate $\mathrm{S} 04 \mathrm{~K}$. The pigment produced by this isolate also showed absorption maxima at 535 $\mathrm{nm}$ (Fig. 1) further indicating it to be prodigiosin. Hence further studies were carried out using this isolate.

Table 2 summarizes the colony morphology and biochemical characteristics of prodigiosin producing

Table 2. Morphological and biochemical characteristics of prodigiosin-producing bacterial isolate S04Ks

\begin{tabular}{|c|c|}
\hline Parameter & Characteristic \\
\hline $\begin{array}{l}\text { Gram nature, motility and } \\
\text { general morphology }\end{array}$ & Gram negative motile short rods \\
\hline Size and color & $1 \mathrm{~mm}$, magenta red \\
\hline Shape and consistency & Circular and smooth \\
\hline Elevation & Raised \\
\hline Opacity & Translucent \\
\hline Margin & Irregular \\
\hline Oxidase & Negative \\
\hline Catalase & Positive \\
\hline $\begin{array}{l}\text { Sugar fermentation with } \\
\text { acid prodction }\end{array}$ & $\begin{array}{l}\text { Maltose, sorbitol, gkucose, } \\
\text { sucrose, inulin, galactose, } \\
\text { dextrin, mannitol }\end{array}$ \\
\hline No fermentation & Glycerine, xylose, starch \\
\hline Positive biochemical tests & $\begin{array}{l}\text { Voges Prausker, lysine } \\
\text { decarboxylase, gelatin hydrolysis, } \\
\text { nitrite reductase }\end{array}$ \\
\hline Negative biochemical tests & $\begin{array}{l}\text { Indole, methyl red, } \\
\text { phenylanaline deaminase }\end{array}$ \\
\hline
\end{tabular}




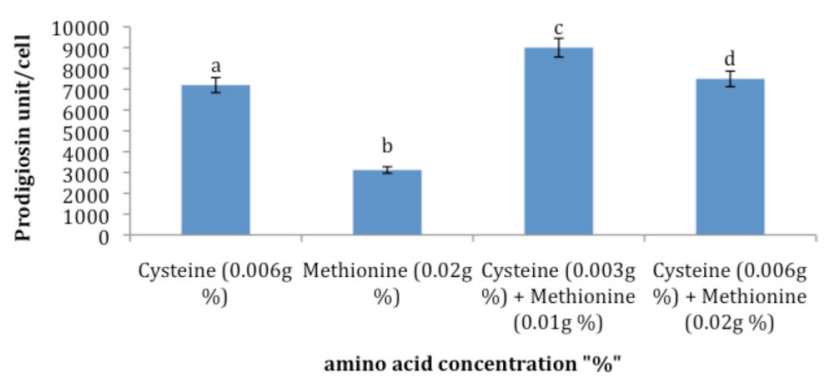

Fig. 2. Effect of $\mathrm{pH}$ on prodigiosin production by S. nematodiphila in nutrient broth. Different letters indicate statistically significant differences between groups (mean $\pm \mathrm{SD}, n=3$, one way ANOVA, $p<0.05)$.

isolate $\mathrm{S} 04 \mathrm{~K}$. This isolate showed $98 \%$ homology to the $S$. nematodiphila strain, as identified by 16s rRNA technique.

Figs. 2 to 5 show the optimized physicochemical parameters for prodigiosin production by S. nematodiphila. isolate $\mathrm{S} 04 \mathrm{~K}$. The differences in mean values represented in these graphs were found to be statistically significant at $p$ $<0.05$. It was observed that $\mathrm{pH} 8$, temperature $25{ }^{\circ} \mathrm{C}, 1 \%$ $\mathrm{NaCl}$ concentration and static conditions were optimal for prodigiosin production when S. nematodiphila was cultivated in NB.

Results from optimization of carbon and phosphate sources for prodigiosin production by S. nematodiphila are shown in Figs. 6 and 7, respectively. The differences in mean values represented in both graphs were found to

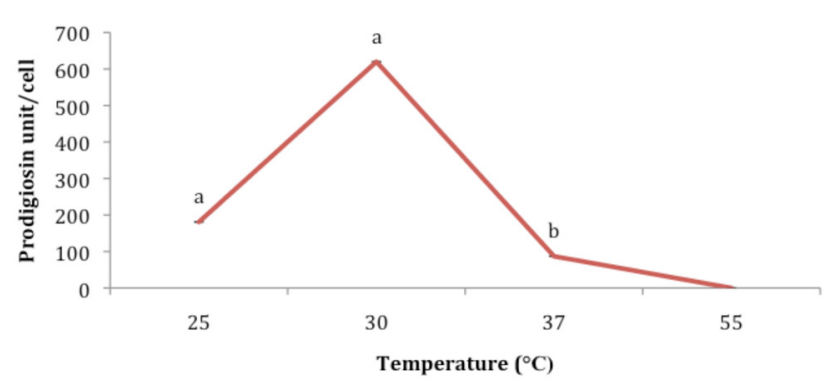

Fig. 4. Effect of $\mathrm{NaCl}$ concentration on prodigiosin production by $S$. nematodiphila in nutrient broth. Different letters indicate statistically significant differences between groups (mean $\pm \mathrm{SD}, n$ $=3$, one way ANOVA, $p<0.05$ ).

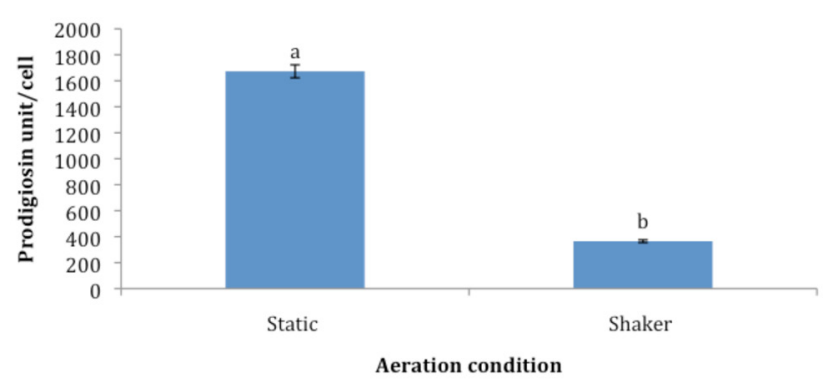

Fig. 6. Effect of carbon sources on prodigiosin production by S. nematodiphila in nutrient broth. Different letters indicate statistically significant differences between groups (mean $\pm \mathrm{SD}, n$ $=3$, one way ANOVA, $p<0.05$ ).

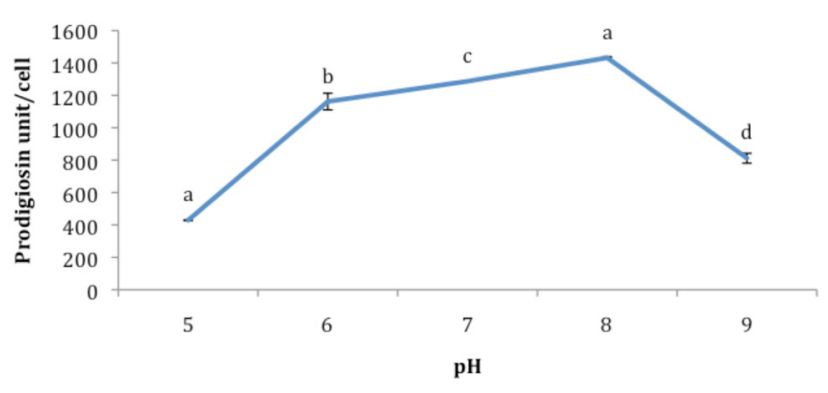

Fig. 3. Effect of temperature on prodigiosin production by $S$. nematodiphila in nutrient broth. Different letters indicate statistically significant differences between groups (mean $\pm \mathrm{SD}, n$ $=3$, one way ANOVA, $p<0.05$ ).

be statistically significant at $p<0.05$. It was observed that addition of $0.2 \%$ mannitol and $0.2 \%$ cobalt phosphate to previously optimized NB medium further improved prodigiosin production by $S$. nematodiphila.

The effect of various amino acids on prodigiosin production was observed qualitatively on NA plates using the disc method. Among the amino acids used, methionine and cysteine did not inhibit prodigiosin production at $0.1 \%$ concentration. Hence optimized concentrations of these amino acids were further determined in our study. Figs. 8 and 9 represent the results for cysteine and methionine concentrations, respectively, required for optimum prodigiosin production. Effect of incorporation of both these amino acids simultaneously in the production

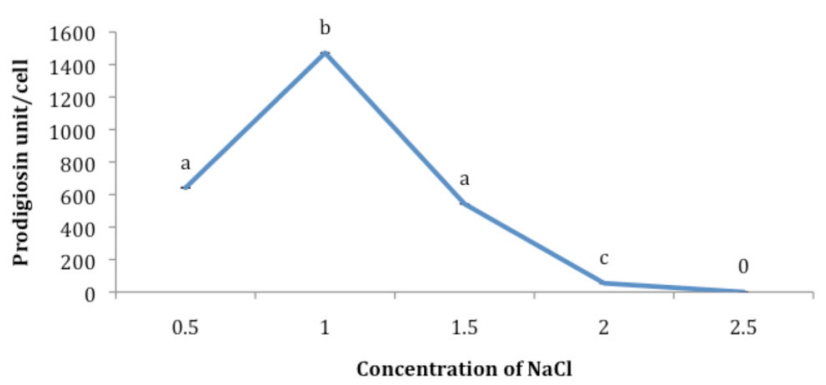

Fig. 5. Effect of aeration condition on prodigiosin production by $S$. nematodiphila in nutrient broth. Different letters indicate statistically significant differences between groups (mean $\pm \mathrm{SD}, n$ $=3$, one way ANOVA, $p<0.05$ ).

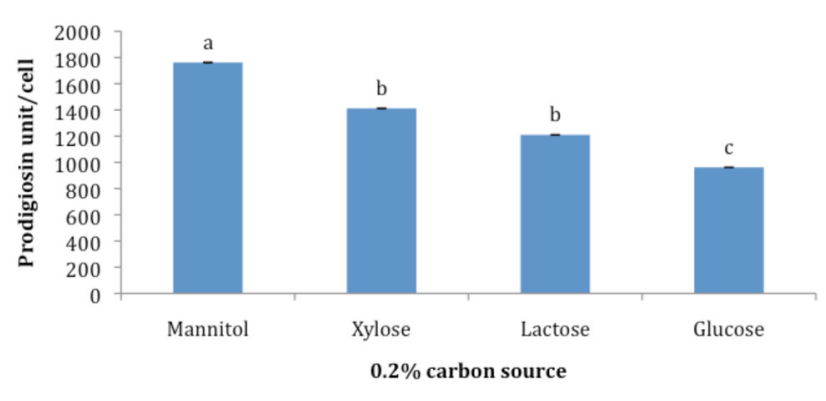

Fig. 7. Effect of phosphate sources on prodigiosin production by $S$. nematodiphila in nutrient broth containing $0.2 \%$ mannitol. Different letters indicate statistically significant differences between groups (mean $\pm \mathrm{SD}, n=3$, one way ANOVA, $p<0.05$ ). 


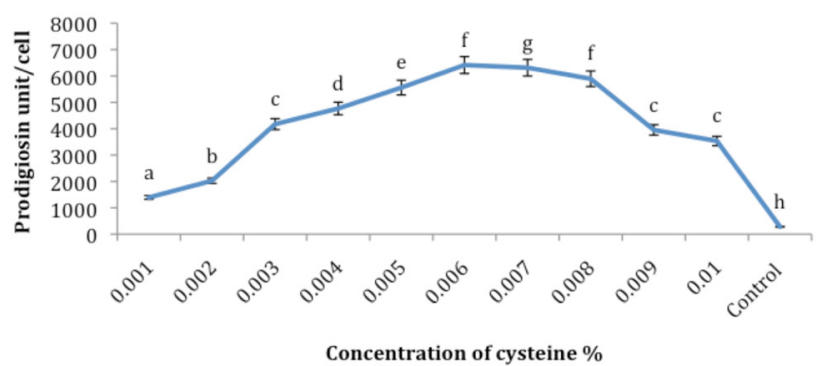

Fig. 8. Effect of concentration of cysteine on prodigiosin production by $S$. nematodiphila in nutrient broth containing $0.2 \%$ mannitol and $0.2 \%$ cobalt phosphate. Different letters indicate statistically significant differences between groups (mean $\pm \mathrm{SD}, n$ $=3$, one way ANOVA, $p<0.05)$.

medium is shown in Fig. 10. The differences in mean values represented in all three graphs were found to be statistically significant at $p<0.05$. It was observed that $0.006 \%$ cysteine and $0.02 \%$ methionine considerably improved prodigiosin production. However, when added together in optimum concentrations, it showed a decrease in prodigiosin production. Better production was obtained when the concentration of both amino acids was reduced by half.

Results from comparison of prodigiosin production in NB, LB and optimized medium is shown in Fig. 11. The differences in mean values represented in this graph were found to be statistically significant at $p<0.05$. The pigment production in optimized media showed 64.78 and $73.23 \%$ increase as compared to standard NB and LB medium, respectively.

\section{Discussion}

The production of prodigiosin is mainly associated with, but not limited to, S. marcescens (Furnster 2003). The cultural and biochemical characterization of cultures remains the gold standard for preliminary identification. Also, these findings are best confirmed with the help of gene sequencing techniques, including 16s rRNA. Although the API system has become increasingly common and widely used for identification of bacteria (Janda, Abbott 2002), it does not

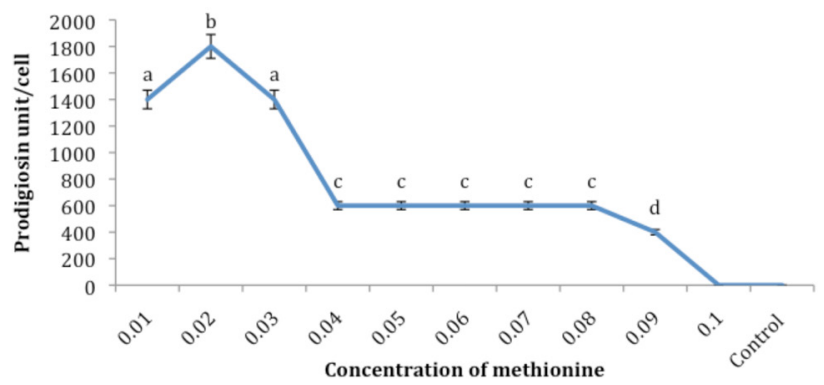

Fig. 10. Effect of concentration of amino acids on prodigiosin production by $S$. nematodiphila in nutrient broth containing $0.2 \%$ mannitol and $0.2 \%$ cobalt phosphate. Different letters indicate statistically significant differences between groups (mean $\pm \mathrm{SD}, n$ $=3$, one way ANOVA, $p<0.05$ ).

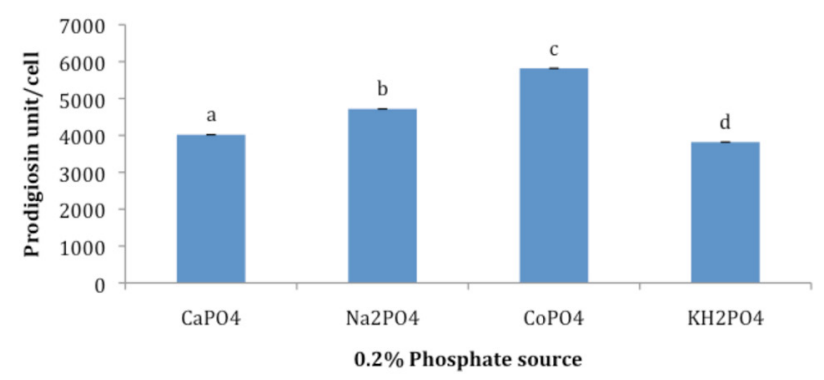

Fig. 9. Effect of concentration of methionine on prodigiosin production by S. nematodiphila in nutrient broth containing $0.2 \%$ mannitol and $0.2 \%$ cobalt phosphate. Different letters indicate statistically significant differences between groups (mean $\pm \mathrm{SD}, n$ $=3$, one way ANOVA, $p<0.05$ ).

show absolute sensitivity in bacterial identification. A study reported correct identification of $85 \%$ of S. marcescens isolates using the API 20E system (Hejazi et al. 1997). This was mainly due to the limited sugar fermentation codes available in this system. For instance, the differentiating characteristic in the identification of S. marcescens and Serratia liquefaciens strains is the fermentation of arabinose. The former is unable to ferment arabinose in peptone water, whereas the latter shows an arabinose positive test. Hence, sugar fermentation tests, including arabinose and raffinose, should ideally be carried out for identification of Serratia sp., irrespective of the API results (Hejazi et al. 1997).

Prodigiosin is a secondary metabolite and typically appears in the later stages of bacterial growth (Harris et al. 2004). Various physicochemical factors critically influence the production of prodigiosin (Williamson et al. 2005). Characteristically, prodigiosin is produced in slightly alkaline culture conditions and low temperatures. In fact, temperatures higher than $37^{\circ} \mathrm{C}$ considerably inhibit prodigiosin production (as observed in the current study). This is one of the main reasons why most of the pathogenic strains of S. marcescens are non-pigmented (Roy et al.2014).

Previously, a differential and selective media i.e., capryllate thallous agar medium, has been used for isolation of Serratia sp. This medium contains caprylate as a carbon source, which is readily utilized by Serratia sp., and thallous

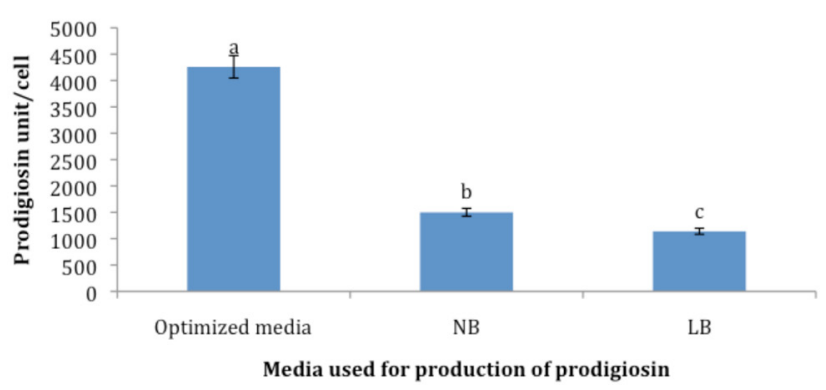

Fig. 11. Comparison of media for prodigiosin production by $S$. nematodiphila. Different letters indicate statistically significant differences between groups (mean $\pm \mathrm{SD}, n=3$, one way ANOVA, $p<0.05)$. 
salts that inhibit other organisms (Staunton, Wilkinson 1997). However, prodigiosin production has found to be ideal in a standard or slightly modified nutrient medium, as compared to other media (Pryce, Terry 2000; Samrot et al. 2011; Pradeep et al. 2013). Hence, NB was used for the optimization of prodigiosin in the present study.

The current study indicated optimum prodigiosin production by $S$. nematodiphila in $\mathrm{NB}(\mathrm{pH}$ 8) containing $1 \% \mathrm{NaCl}$, on incubation at $25{ }^{\circ} \mathrm{C}$ for $48 \mathrm{~h}$ under static conditions. Similar to our findings, the optimum $\mathrm{pH}$ for prodigiosin production was reported to be between 8 and 8.5 by S. marcescens (Sole et al. 1994). They further reported that the prodigiosin production was independent of initial $\mathrm{pH}$ in un-buffered medium. The variations in prodigiosin production were noted only when buffers were used in cultures of non-proliferating cells. A recent study on optimization of parameters for production of prodigiosin by $S$. nematodiphila RL2 reported maximum production at $35{ }^{\circ} \mathrm{C}$ in 46 to $48 \mathrm{~h}$ in nutrient broth medium ( $\mathrm{pH} 6$ to 7) supplemented with $1 \%$ lactose, yeast extract and uranyl acetate (Gondil et al. 2017). However, pH 7, inoculum concentration $5 \%$, temperature $30{ }^{\circ} \mathrm{C}$ and incubation period of $36 \mathrm{~h}$ were reported to be optimum for prodigiosin production by S. marcescens MBB05 (Pradeep et al. 2013). Similarly, the optimal condition for pigment production by another S. marcescens strain was found to be in NB (pH 7) at $28^{\circ} \mathrm{C}$ after $72 \mathrm{~h}$ (Samrot et al. 2011).

In contrast to our observation, another study reported optimum production of prodigiosin under aeration conditions (Heinemann et al. 1970). Similarly, shaker conditions (150 rpm) and a lower concentration of $\mathrm{NaCl}$ $(0.4 \%)$ were found to be ideal for prodigiosin production by another strain of $S$. marcescens (Vijayalakshmi, Jagathy 2016). The optimum temperature $\left(25^{\circ} \mathrm{C}\right)$ and incubation time $(30 \mathrm{~h})$, however, were similar to those observed in our study. A halophilic Serratia sp. BTWJ8 has been also reported to grow and produce prodigiosin at up to 5.8\% $\mathrm{NaCl}$ concentration (Krishna 2008).

Apart from physicochemical and growth conditions, nutritional parameters including carbon and nitrogen sources, micronutrients and amino acids are also important for production of secondary metabolites in bacteria. In the current study, $0.2 \%$ mannitol, $0.2 \%$ cobalt phosphate, $0.006 \%$ cysteine and $0.02 \%$ methionine considerably improved prodigiosin production.

In a previous study, $2 \%$ sodium oleate resulted in optimum prodigiosin production by $S$. marcescens (Khanafari et al. 2006). Another study reported 1.5\% dextrose, $1 \%$ yeast extract to be ideal carbon and nitrogen sources. However, maximum pigment production was observed using $5 \%$ peanut oil cake (Vijayalakshmi, Jagathy 2016). An interesting observation was reported by Giri et al (2004). In NB, the maximum prodigiosin production was obtained at 28 and $30^{\circ} \mathrm{C}$, and it was completely inhibited at $37^{\circ} \mathrm{C}$. However, when powdered peanut broth was used as a carbon source for prodigiosin production by $S$. marcescens, pigment production was observed even at $37^{\circ} \mathrm{C}$. Moreover, it was equal to the amount of pigment produced at $30^{\circ} \mathrm{C}$ in NB. In another study, powdered peanut seed broth at 37.6 $\mathrm{mg} \mathrm{mL}^{-1}$ and powdered sesame seed broth at $16.5 \mathrm{mg} \mathrm{mL}^{-1}$ showed optimized pigment production by $S$. marcescens, as compared to nutrient broth with concentration $0.51 \mathrm{mg}$ $\mathrm{mL}^{-1}$ (Shahitha, Poornima 2012).

In our study, the presence of nitrogen sources supported growth of culture but pigment production was very low as compared to non-optimised NB media. This may be because NB contains peptone as a nitrogen source, and further addition of other nitrogen sources may have a negative effect on pigment production. Hence optimization of nitrogen sources was not carried out in our study. In another study, $0.4 \%$ ram horn peptone along with mannitol showed maximum production of prodigiosin by $S$. marcescens after $48 \mathrm{~h}$ (Kurbanoglu et al. 2015). S. marcescens MN5 also showed enhanced production of prodigiosin at $22{ }^{\circ} \mathrm{C}$ and $\mathrm{pH} 9$ on the addition of $1 \%(\mathrm{w} / \mathrm{v})$ peptone and crude glycerol as nitrogen and carbon sources, respectively (Elkenawy et al.2017). The presence of inorganic phosphates effectively increased the synthesis of prodigiosin by nonproliferating cells of $S$. marcescens at a concentration of $0.3 \mathrm{mM}$ (Whitney et al. 1977). Other studies also reported positive effects of diammonium phosphate (Sumathi et al. 2014) and mono- and di-potassium phosphate (Domrose et al. 2015) on prodgiosin production. In addition, phosphates have also been reported to play an important role in biosynthesis of prodigiosin in Serratia sp. (Slater et al. 2003).

Although low concentrations of amino acids positively influence prodigiosin production, higher concentrations are associated with inhibition of pigment production. This is probably due to the diversion of excess amino acids to act as repressors in prodigiosin biosynthesis pathways (Qadri et al. 1973). This was further supported by a study carried out by incorporating both amino acids in growth media, and studying their effect on prodigiosin production (Fig. 10). Interestingly, increased production of prodigiosin was obtained only when the concentrations of both amino acids were reduced by half. This indicates that prodigiosin production is dependent on specific amino acids, as well as the total concentration of different amino acids. Similar to our findings, another study also reported methionine and cysteine to effectively increase prodigiosin production at 0.01 and $0.03 \%$ concentration, respectively, in presence of $0.4 \%$ mannose and $0.1 \% \mathrm{NaCl}$ in $\mathrm{M} 9$ medium ( $\mathrm{pH} 8$ ) at $28^{\circ} \mathrm{C}$ in $24 \mathrm{~h}$ (Bharmal et al. 2012). Key roles of other amino acids i.e. thiamine, alanine, histidine and proline in the formation of the monopyrrole moiety of prodigiosin were also suggested (Goldschmitt, Williams 1968; Lim et al. 1976). The incorporation of proline in the biosynthetic pathway of prodigiosin was suggested (Scott et al. 1976).

The basic aim of optimization studies is to improve the 
metabolite yield by bacteria. In the current study, 64.78 and $73.23 \%$ increase in prodigiosin production was observed as compared to standard NB and LB medium, respectively. Similarly, a $65 \%$ increase in prodigiosin production by $S$. nematodiphila RL2 in optimized medium as compared to nutrient broth was reported (Gondil et al. 2017). Considerable increase (over three times) in prodigiosin production by $S$. marcescens strain MSK1 has also been reported in optimized M9 medium (Bharmal et al. 2012). Another study reported over 1.5 to 3 times increase in prodigiosin production in optimized media containing casein rich broth and vegetable oils, as compared to nutrient broth and glycerol yeast broth (Parani, Saha 2008).

\section{Conclusions}

Prodigiosin production in Serratia sp. is dependent on nutritional as well as physicochemical parameters that are unique to every strain isolated from the environment. For instance, methionine and cysteine do not necessarily induce or improve pigment production in other strains of $S$. nematodiphila. Also, the amino acids selected in our study, on the basis of literature survey, did not support prodigiosin production by S. nematodiphila. This suggests that either a novel derivative of prodigiosin may be produced by the test strain, or that it follows an unique biosynthesis pathway for prodigiosin production. Further studies are hence required to study the biosynthetic pathway of prodigiosin by S. nematodiphila to obtain more insights into the intermediates of the pathway that will help us in biochemical characterization of the pigment. The optimized media showed over $60 \%$ increase in prodigiosin production in our study, indicating good potential of the test isolate for application in industries that use a sustainable approach for production of raw materials.

\section{References}

Babu S., Shenolikar I.S. 1995. Health and nutritional implications of food colors. Indian J. Med. Res. 102: 245-249.

Bharmal M.H., Jahagirdar N.,Aruna K.2012.Study on optimization of prodigiosin production by Serratia marcescens MSK1 isolated from air. Int. J. Adv. Biol. Res. 2: 671-680.

Cang S., Sanada M., Johdo O., Ohta S., Nagamatsu Y., Yoshimoto A. 2000. High production of prodigiosin by Serratia marcescens grown on ethanol. Biotechnol. Lett. 22: 1761-1765.

Darshan N., Manonmani H.K. 2015. Prodigiosin and its potential applications. J. Food Sci. Technol. 52: 5393-5407.

Domröse A., Klein, A.S., Hage- Hülsmann J., Thies S., Svensson V., Classen T., Pietruszka J., Jaeger K.E., Drepper T., Loeschcke A. 2015. Efficient recombinant production of prodigiosin in Pseudomonas putida. Front. Microbiol. 6: 972.

Dufosse L. 2009. Pigments, Microbial. In: Encyclopedia of Microbiology. $3^{\text {rd }}$ Ed. Elsevier/Academic Press, New York, pp. 457-471.

Duran N., Teixeira M.F.S., Conti R., Esposito E. 2002. Ecologicalfriendly pigments from fungi. Crit. Rev. Food Sci. 42: 53-66.

Elkenawy N.M., Yassin A.S., Elhifnawy H.N., Amin M.A.
2017. Optimization of prodigiosin production by Serratia marcescens using crude glycerol and enhancing production using gamma radiation. Biotechnol. Rep. 14: 47-53.

Furstner A. 2003. Chemistry and biology of roseophilin and the prodigiosin alkaloids: a survey of the last 2500 years. Angew Chem. Int. Ed. Engl. 42: 3582-3603.

Gerber N.N., Lechevalier M.P.1976. Prodiginine (prodigiosin-like) pigments from streptomyces and other aerobic actinomycetes. Can. J. Microbiol. 22: 658-667.

Giri A.V., Anandkumar N., Muthukumaran G., Pennathur G. 2004. A novel medium for the enhanced cell growth and production of prodigiosin from Serratia marcescens isolated from soil. BMC Microbiol. 4: 11.

Goldschmitt M.C., Williams R.P. 1968. Thiamine induced formation of the monopyrrole moiety of prodigiosin. J. Bacteriol. 96: 609-616.

Gondil V.S., Asif M., Bhalla T.C. 2017. Optimization of physicochemical parameters influencing the production of prodigiosin from Serratia nematodiphila RL2 and exploring its antibacterial activity. 3 Biotech. 7: 338 .

Hardjito L., Huq A., Colwell R.R. 2002. The influence of environmental conditions on the production of pigment by Serratia marcescens. Biotechnol. Bioprocess Eng. 7: 100-104.

Harris A.K.P., Williamson N.R., Slater H., Cox A., Abbasi S., Foulds I., Simonsen H.T., Leeper F.J., Salmond G.P.C. 2004. The Serratia gene cluster encoding biosynthesis of the red antibiotic, prodigiosin, shows species and strain dependent genome context variation. Microbiology 150: 3547-3560.

Heinemann B., Howard A.J., Palocz H.J. 1970. Influence of dissolved oxygen levels on production of L-asparaginase and prodigiosin by Serratia marcescens. Appl. Microbiol. 19: 800804.

Hejazi A., Falkiner F.R. 1997. Serratia marcescens. J. Med. Microbiol. 46: 903-912.

Hobson D.K., Wales D.S. 1998. Green dyes. J. Soc. Dyers Colour 114: 42-44.

Holt J.G., Krieg N.R., Sneath P.H.A, Staley J.T, Williams S.T. 1994. Bergey's Manual of Determinative Bacteriology. $9^{\text {th }}$ Ed. Williams \& Wilkins, Baltimore.

Janda J.M., Abbott S.L. 2002. Bacterial identification for publication: when is enough enough? J. Clin. Microbiol. 40: 1887-1891.

Khanafari A., Assadi M.M., Fakhr F.A. 2006. Review of prodigiosin, pigmentation in Serratia marcescens. Online J. Biol. Sci. 6: 1-13.

Kim C.H., Kim S.W., Hong S.I. 1999. An integrated fermentation separation process for the production of red pigment by Serratia sp. KH-95. Process Biochem. 35: 485-490.

Kobylewski S., Jacobson M.F. 2012. Toxicology of food dyes. Int. J. Ocupp. Environ. Health 18: 220-246.

Krishna, J.G., 2008. Pigment production by marine Serratia sp. BTW J8, Ph.D Thesis, Microbial Technology Laboratory, Department of Biotechnology, Cochin University of Science and Technology, Cochin. India.

Kumar M., Venkatesh M.P., Kumar T.M.P. 2017. Colourants and additives: Existing and emerging safety concerns. Int. J. Pharm. Clin. Res. 9: 525-533.

Kurbanoglu E.B., Ozdal M., Ozdal O.G., Algur O.F. 2015.Enhanced production of prodigiosin by Serratia marcescens MO-1 using ram horn peptone. Braz. J. Microbiol. 46: 631-637.

Kurbanoglu E.B., Ozdal M., Ozdal O.G.,Algur O.F.2015.Enhanced production of prodigiosin by Serratia marcescens MO-1 using ram horn peptone. Braz. J. Microbiol. 46: 631-637. 
Lawanson A.O., Sholeye F.O. 1976. Inhibition of prodigiosin formation in Serratia marcescens by adenosine triphosphate. Experientia 32: 439-440.

Lewis S.M., Corpe W.A. 1964. Prodigiosin producing bacteria from marine sources. Appl. Microbiol. 12: 13-17.

Lim D.V., Qadri S.M., Nichols C., Williams R.P. 1977. Biosynthesis of prodigiosin by non-proliferating wild-type Serratia marcescens and mutants deficient in catabolism of alanine, histidine, and proline. J. Bacteriol. 129: 124-130.

Lim D.V., Qadri S.M., Williams R.P. 1976. Incorporation of proline into prodigiosin by a put mutant of Serratia marcescens. Appl. Environ. Microbiol. 31: 738-742.

Narsing Rao M.P., Xiao M., Li W.J. 2017. Fungal and bacterial pigments: secondary metabolites with wide applications. Front Microbiol. 8: 1113.

Parani K., Saha B.K. 2008. Optimization of prodigiosin production from a strain of Serratia marcescens SRI and screening for antifungal activity. J. Biol. Contr. 22: 73-79.

Pradeep B.V., Pradeep F.S., Palaniswamy M. 2013. Optimization and production of prodigiosin from Serratia marcescens MBB05 using various natural substrates. Asian J. Pharm. Clin. Res. 6: 34-41.

Pryce L.H., Terry F.W. 2000. Spectrophotometric assay of gene expression: Serratia marcescens pigmentation. Bioscene 26: 3-13.

Purnama H., Hidayati N., Safitri D.S., Rahmawati S. 2017. Effect of initial treatment in the preparation of natural indigo dye from Indigofera tinctoria. AIP Conference Proceedings 1855: 020022.

Qadri H.S.M., Williams R.P. 1972. Induction of prodigiosin biosynthesis after shift-down in temperature of nonproliferating cells of Serratia marcescens. Appl. Microbiol. 23: 704-709.

Qadri H.S.M., Williams R.P. 1973. Role of methionine in biosynthesis of prodigiosin by Serratia marcescens. J. Bacteriol. 116: 1191-1198.

Rjazantseva I.N., Andreeva I.N., Ogorodnikova T.I. 1994. Effect of various growth conditions on pigmentation of Serratia marcescens. Microbios 79: 155-161.

Roy P., Ahmed N.H., Grover R.K. 2014. Non-pigmented strain of Serratia marcescens: an unusual pathogen causing pulmonary infection in a patient with malignancy. J. Clin. Diagn. Res. 8: 5-6.

Samrot A.V., Chandana K., Senthilkumar P., Kumar N. 2011. Optimization of prodigiosin production by Serratia marcescens SU-10 and evaluation of its bioactivity. Int. Res. J. Biotechnol. 2: 128-133.

Scott R.H., Hussain Qadri S.M., Williams R.P. 1976. Role of L-proline in the biosynthesis of prodigiosin. Appl. Environ. Microbiol. 32: 561-566.

Shahitha S. Poornima K. 2012. Enhanced production of prodigiosin production in Serratia marcescens. J. Appl. Pharm.
Sci. 2: 138-140.

Silverman M.P., Munoz E.F. 1973. Effect of iron and salt on prodigiosin synthesis in Serratia marcescens. J. Bacteriol. 114: 999-1006.

Slater H., Crow M., Everson L., Salmond G. 2003. Phosphate availability regulates biosynthesis of two antibiotics, prodigiosin and carbapenem, in Serratia via both quorumsensing-dependent and-independent pathways. Mol. Microbiol. 47: 303-320.

Sole M., Rius N., Francia A., Loren J.G. 1994. The effect of pH on prodigiosin production by non-proliferating cells of Serratia marcescens. Lett. Appl. Microbiol. 19: 341-344.

Stange S., Steudler S., Delenk H., Werner A., Walther T., Wagenführ A. 2019. Influence of the nutrients on the biomass and pigment production of Chlorociboria aeruginascens. J. Fungi 5: 40.

Staunton J., Wilkinson B. 1997. Biosynthesis of erythromycin and rapamycin. Chem. Rev. 97: 2611-2630.

Sumathi C., Priya M.P., Swarnalatha S., Dinesh M.G., Sekaran G. 2014. Production of prodigiosin using tannery fleshing and evaluating its pharmacological effects. Sci. World J. 2014: 290327.

Venil C.K., Lakshmanaperumalsamy P. 2009. An insightful overview on microbial pigment, prodigiosin. Electr. J. Biol. 5: 49-61.

Vijayalakshmi K., Jagathy K. 2016. Production of prodigiosin from Serratia marcescens and its antioxidant and anticancer potential. Int. J. Adv. Res. Biol. Sci. 3: 75-88.

Wei Y.H., Chen W.C. 2005. Enhanced production of prodigiosinlike pigment from Serratia marcescens $\mathrm{SM} \Delta \mathrm{R}$ by medium improvement and oil supplementation strategies. J. Biosci. Bioeng. 99: 616-622.

Wei Y.H., Yu W.J., Chen W.C. 2005. Enhanced undecyl prodigiosin production from Serratia marcescens SS-1 by medium formulation and amino-acid supplementation. J. Biosci. Bioeng. 100: 466-471.

Williams R.P., Green J.A. 1956. Studies on pigmentation of Serratia marcescens III. J. Bacteriol. 72: 537-542.

Williamson N.R., Simonsen H.T., Ahmed R.A., Goldet G., Slater H., Woodley L., Leeper F.J., Salmond G.P. 2005. Biosynthesis of the red antibiotic, prodigiosin, in Serratia: identification of a novel 2-methyl-3-n-amyl-pyrrole (MAP) assembly pathway, definition of the terminal condensing enzyme, and implications for undecylprodigiosin biosynthesis in Streptomyces. Mol. Microbiol. 56: 971-989.

Witney F.R., Failia M.L., Weinberg E.D. 1977. Phosphate inhibition of secondary metabolism in Serratia marcescens. Appl. Environ. Microbiol. 33: 1042-1046.

Yusuf M., Shabbir M., Mohammed F. 2017. Natural colorants: historical, processing and sustainable prospects. Nat. Prod. Bioprospect. 7: 123-145. 